\title{
The Tension and Temperature Automatic Control Device for the Cutting Wire of EPS Cutting Machine
}

\author{
Bin Liu ${ }^{1, a}$, Hongyan Jü, b , Zhongwei Zhao, c , Gong He ${ }^{1, d^{*}}$ and Haibin Wang ${ }^{2, \mathrm{e}}$ \\ ${ }^{1}$ Information \&Technology College, Jilin Agriculture University, Changchun 130118, China \\ ${ }^{2}$ Jilin Tobacco Industry Co., Ltd, Yanji 133000, China \\ aliubin062690@163.com, bdidiaoxiaoju@163.com, c1411864225@qq.com, \\ dgonghe@jlau.edu.cn, ${ }^{\mathrm{e}} 5064426 @ q q . c o m$
}

\begin{abstract}
Keywords: Expanded polystyrene, Automatic control, Tension, Temperature
Abstract. Expanded polystyrene(EPS) is a new type of thermal insulation material with excellent properties. It is widely used in the field of external wall thermal insulation, packaging, advertising and others. It has become a hot spot in the field of plate processing that how to optimize EPS board line cutting process. This paper designs a kind of device with automatic control of the tension and temperature of cutting wire based on sensor technology and automatic control technology. According to the preset tension, the precisely adjustment can be done by the control system. The device provides a stable power by voltage controlled constant current source supplying for cutting wire so that it can keeps constantly the temperature in the best range. The device not only solves the problem of low accuracy caused by artificial adjustment and avoids the security risks of operation, but also improves the quality and efficiency of cutting.
\end{abstract}

\section{Introduction}

The most widely used thermal insulation materials in building were EPS and XPS. The rate of annual demands grew constantly, expecially EPS. And the processing enterprises of EPS became the emerging building industry. National $12^{\text {th }}$ Five-Year program revised further the policy of energy saving and emission reduction, which raised higher requirements for building energy efficiency. The insulation materials, which were required by "External wall thermal insulation construction technology conditions" released by Beijing Construction Committee, mainly refers to EPS foam board[1].

The processing technology of EPS foam board mostly applied line cutting at present, whose quality and efficiency of cutting was largely affected by the tension and temperature of the cutting wire. In the cutting process, low tension resulted in the uneven flatness of EPS foam board, conversely, high tension easily led to broken cutting wire if the speed of was too fast and caused production efficiency decreasing and rejection rate raising. In terms of temperature, when the cutting speed was constant, the incision would be too large to meeting requirements if the temperature was too high, however, the low temperature contributed to tough cutting and broken cutting wire[2]. In the profile cutting process, there were correlation between temperature and speed of cutting and it might lead to overcut phenomenon at the corner if temperature couldn't be adjusted.

The types of cutting machine for EPS material were mainly ordinary plate cutting machine, CNC decoration line cutting machine and CNC profile cutting machine[3]. The ordinary plate cutting machine existed the problem such as simple mechanical structure, single function and low accuracy and efficiency, and the machine was only applied in the standard block board cutting. The CNC decoration line cutting machine mostly adopted general gantry type structure on the market, and mainly used in the eaves line and feet line of the building and the dovetail of board. The work principle of CNC profile cutting machine was similar to the lathe, the machine could cut 3D shape such as cone or sphere[4]. 
For any line cutting machine, there were three methods to adjust the tension of cutting wire in the domestic at present: the string type, nut type and pull type adjustment[5]. No matter what kind of methods depends on artificial experience to complete adjustment. The cutting wires adjusted artificially couldn't keeping consistent, leading to the difference of accuracy of each cutting wire and unable to make the cutting speed to be optimized, causing the reduction of cutting efficiency. And in the process of artificial adjustment of cutting wire tension, the wire might break because of high tension.

\section{Overall Framework}

The whole device can be divided into two parts, the tension adjustment of cutting wire and the temperature adjustment of cutting wire. The former includes the cutting wire position confirmation and the tension adjustment, and the latter includes the constant current source power supply and the temperature detection. The whole system's block diagram is shown as Fig. 1.

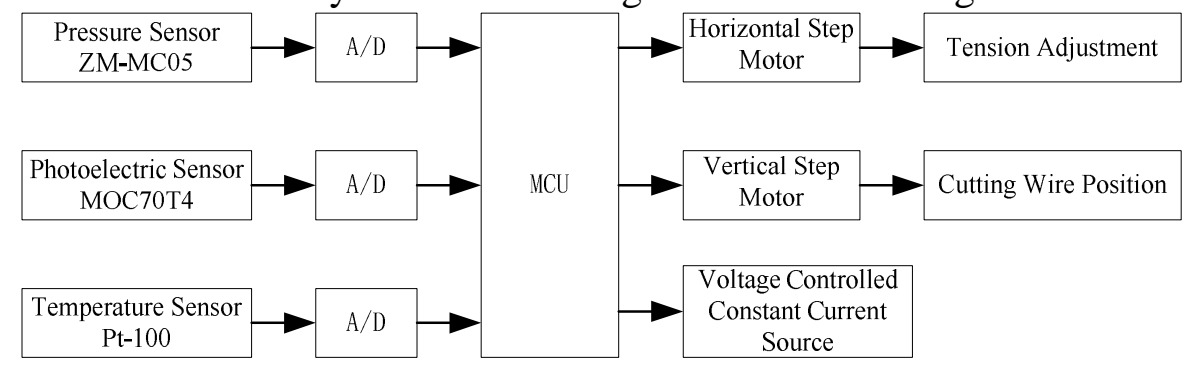

Fig. 1 the Block Diagram of the Device

The device design model is shown as Fig. 2. The tension of EPS cutting wire adjustment is based on the principle of vertical measurement and precision adjustment, and applies pressure sensor as signal acquisition equipment. When the device starts working, the vertical stepper motor drives the screw moving down. The photoelectric sensor below the pressure sensor will confirm the initial position of the cutting wire, and when the cutting wire is detected, the pressure sensor measures and transfer the message of the tension of cutting wire to MCU. And then MCU control the horizontal step motor working to make the tension reaching the default value. The cutting wire is powered by the constant current source at the end of cutting wire, the temperature sensor at the same position measures the temperature constantly.

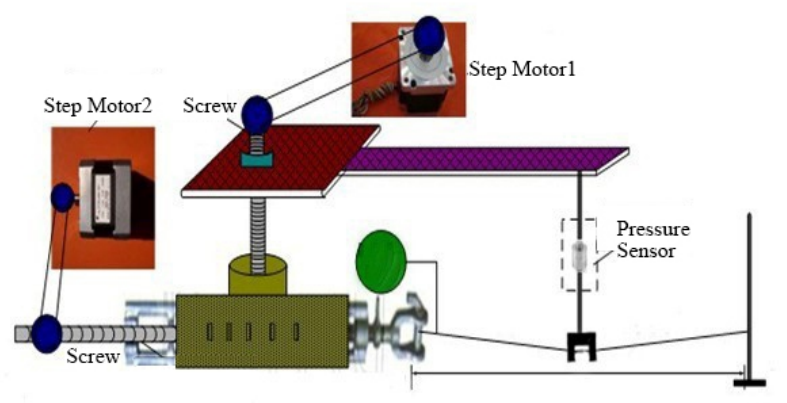

Figure. 2 the Device Design Model

\section{Hardware Design}

The hardware system mainly consists of tension of the cutting wire adjustment circuit and temperature of the cutting wire adjustment circuit.

Tension of the Cutting Wire Adjustment Circuit. This part uses 5V operation for the power, and applies MOC70T4 photoelectric sensor to measuring the position of cutting wire[6]. The sensor has advantages such as easy to install, high reliability and quick response. The sensor is based on the principle of the detected object to block the light, then the synchronous circuit is selected and detect 
whether the object is detected or not[7]. The system uses ZM-MC05 pressure sensor measuring the tension of cutting wire. The circuit schematic is shown as Fig. 4.

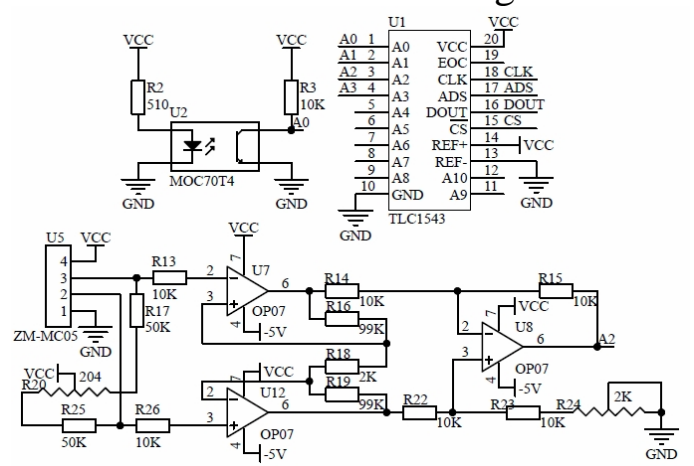

Fig. 3 the Tension Adjustment of Cutting Wire Circuit Schematic

Temperature of the Cutting Wire Adjustment Circuit. The system applies Pt100 temperature sensor to measuring the temperature of cutting wire. The system applies $12 \mathrm{~V}$ voltage controlled constant current source to heating the cutting wire. This circuit converts the digital voltage to analog voltage, and control the conduction angle of IGBT-MOSFET achieving control of output voltage. The voltage signal from the output terminal is fed to the comparator, which makes the current constant. And OP37 precision operational Amplifier is applied to adjusting the voltage signal. The voltage controlled constant current source schematic is shown as Fig. 5.

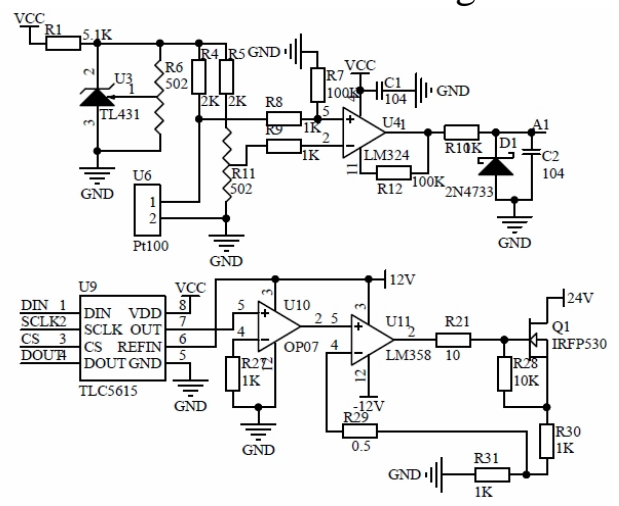

Fig. 4 the Pt100 Temperature Measurement Circuit Schematic

\section{Software Design}

The soft design for hardware is mainly divided into two parts: temperature of the cutting wire adjustment and tension of the cutting wire adjustment.

Temperature of the Cutting Wire Adjustment. The operation process of temperature of the cutting wire adjustment is shown as Fig. 6.

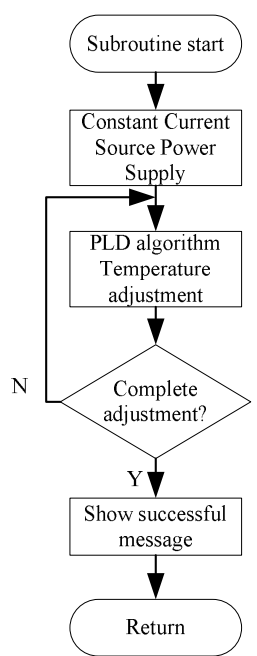

Fig. 5 the Temperature of Cutting Wire Adjustment Program Flow Chat 
Tension of the Cutting Wire Adjustment. The operation process of tension of the cutting wire adjustment is described like thus: firstly, starting the initialization including MCU and relatend modules, then adjustting tension of the cutting wire to preset value by PID algorithm. And there will show the successful massage after adjustment is completed. The flow chart is shown as Fig. 7.

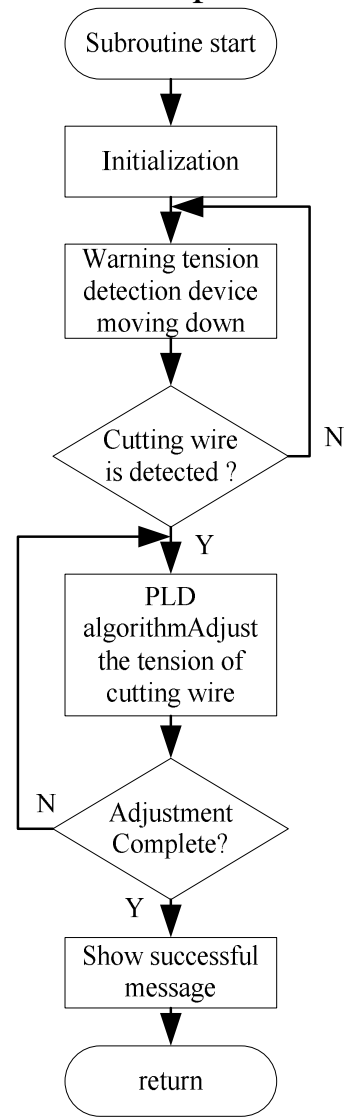

Fig. 6 the Tension of the Cutting Wire Adjustment Program Flow Chart

\section{Conclusion}

\section{The Experimental Condition}

Material of cutting wire: Nichrome wire

Diameter of cutting wire: $0.4 \mathrm{~mm}$

Length of cutting wire: $1.2 \mathrm{~m}$

Density of EPS board: $0.02 \mathrm{~g} / \mathrm{cm}^{3}$

The tools of experiment: $117 \mathrm{c}$ type universal meter, $0^{\circ} \mathrm{C}-300^{\circ} \mathrm{C}$ digital thermometer, digital oscilloscope, $220 \mathrm{~V}$ power supply, $+5 \mathrm{~V} /+12 \mathrm{~V}$ regulated power supply.

Analysis of Detection Data of the Cutting Wire Position. The changing curve of the falling distance of the vertical step motor and the output voltage of the photoelectric sensor is shown as Fig. 11.

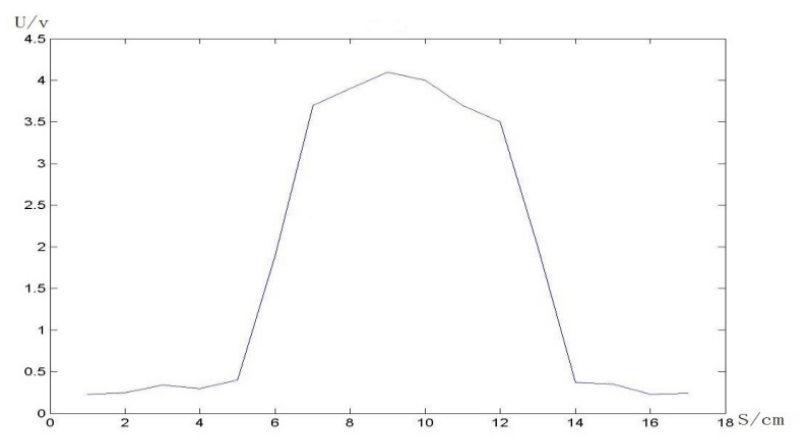

Fig. 7 the Changing Curve of falling distance and photoelectric sensor 
From the changing curve above, the conclusion can be drawn that the central position can be confirmed from where the vertical step motor falls $9 \mathrm{~cm}$.

Analysis of Detection Data of the Cutting Wire Warning Tension. There is conducting data detection of the warning tension of pressure sensor measuring and the displacement distance of the vertical step motor, the detection data is shown as Fig. 12.

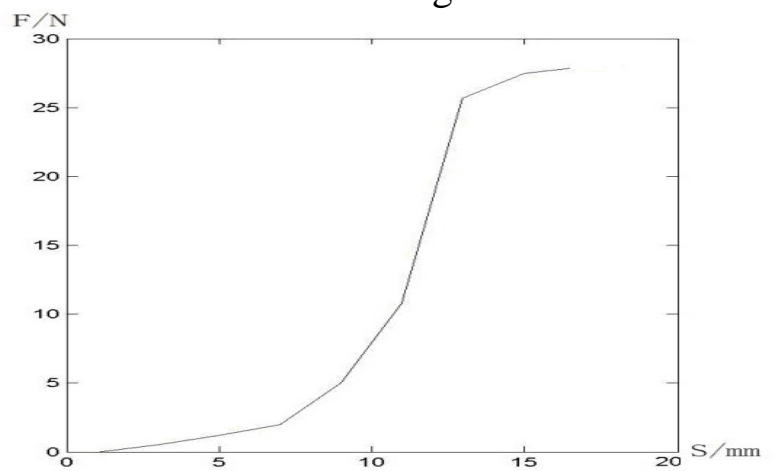

Fig. 8 Relation of Warning Force to Displacement Diagram

The pressure detected by the pressure sensor and displacement distance of the step motor grows exponentially in a certain range. And in this data, when the vertical step motor is falling about $18 \mathrm{~mm}$, the cutting wire reaches the preset warning tension $17.5 \mathrm{~N}$. When the step motor continues falling, the cutting wire breaks.

\section{Acknowledgements}

This work was financially supported by Changchun Science and Technology Bureau(13KG71), Jilin Education Department, Jilin Science and Technology Department(20160623016TC) and Jilin Agricultural University Science and Technology Innovation Fund Project: the tension and temperature automatic control device for the cutting machine and the position of cutting wires.

\section{References}

[1] M.L. Zhou, B.Q. Zhao: Shanghai Building Materials, (2008), No.02, p. 15-16.

[2] H. Liu, J.Z. Fan: Journal of Electric Power, (2000), No.04, p. 257-260.

[3] Y. Li, Y.C. Zhang: Plastics, (2001), No.04, p. 23-28.

[4] C. Du, J. Yang: Journal of Southeast University(Natural Science Edition), (2001), No.03, p. 138-142.

[5] Z.Q. Li: Opto-Electronic Engineering, (1984), No.02, p. 90-94.

[6] G.F. Wang: Maschinen Markt, (2002), No.19, p. 66-68.

[7] H.Q. Sheng, S.Y. Wang: Mechanical \& Electrical Engineering Technology, (2006), No.06, p. $47-50+114$.

[8] H.B. Xu, Y.R. Chen, L. Zhang: Technology Supervision in Petroleum Industry, (1995), No.11, p. 25-27. 